\title{
Notable ampliación del rango altitudinal de Eira barbara Cabeza de Mate (Mammalia: Mustelidae)
}

\author{
Carolina Reyes-Puig, ${ }^{1,2 *}$, Gorki Ríos-Alvear², Juan Pablo Reyes-Puig ${ }^{1,2,3}$ \\ ${ }^{I}$ Museo Ecuatoriano de Ciencias Naturales del Instituto Nacional de Biodiversidad. Rumipamba 341 y Av. De los Shyris. Casilla postal 17-07-8976, \\ Quito, Ecuador \\ ${ }^{2}$ Fundación Oscar Efrén Reyes, Departamento de Ambiente, calle 12 de Noviembre $N^{\circ} 270$ y calle Luis A. Martínez, Baños, Ecuador \\ ${ }^{3}$ Fundación Ecominga-Red de Protección de Bosques Amenazados, Baños, Ecuador \\ *Autor por correspondencia, carolina.reyes@ambiente.gob.ec
}

Editado por/Edited by: Diego F. Cisneros-Heredia, PhD.(c)

Recibido/Received: 2015/04/07. Aceptado/Accepted: 2015/05/12

Publicado en línea/Published online: 2015/05/22. Impreso/Printed: 2015/06/01

\section{Remarkable altitudinal range extension of Eira barbara Tayra (Mammalia: Mustelidae)}

\begin{abstract}
Eira barbara Tayra has been reported across its geographic range from elevations between 0-2400 $\mathrm{m}$, being rare above $1200 \mathrm{~m}$. We report four records of $E$. barbara above $2600 \mathrm{~m}$, obtained by using camera trapping. These records come from Reserva Natural Chamanapamba and Bosque Protector Cerro Candelaria, both located on the province of Tungurahua, eastern slopes of the Andes of Ecuador.

Keywords: Carnivora, Mustelidae, Eira barbara, Tayra, Candelaria, Chamanapamba, Tungurahua, camera trapping, distribution, elevation.

\section{Resumen}

Eira barbara Cabeza de Mate se ha reportado a lo largo de su rango geográfico a elevaciones entre 0-2400 m, siendo raro sobre $1200 \mathrm{~m}$. Reportamos cuatros registros de E. barbara sobre los $2600 \mathrm{~m}$, obtenidos por medio de trampas cámaras. Los registros vienen de la Reserva Natural Chamanapamba y del Bosque Protector Cerro Candelaria, ambos localizados en la provincia de Tungurahua, estribaciones orientales de los Andes de Ecuador

Palabras clave: Carnivora, Mustelidae, Eira barbara, Cabeza de Mate, Candelaria, Chamanapamba, distribución, elevación, trampas cámara.
\end{abstract}

Eira barbara Cabeza de Mate o Tayra es un mamífero carnívoro perteneciente a la familia Mustelidae [1]. Habita desde el centro de México a lo largo de Centroamérica hasta el norte de Argentina, con un rango altitudinal reportado entre 0 y $2400 \mathrm{~m}$ de altitud, pero considerada rara sobre los 1200 metros de elevación [2-4]. En Ecuador, E. barbara habita los bosques de tierras bajas y piemontanos occidentales y orientales, entre los 0 y 1550 m [5-6], con un registro a 2100 metros de elevación [7]. En esta publicación presentamos nuevos registros de $E$. barbara que extienden notablemente el límite superior del rango altitudinal de la especie.

Instalamos ocho estaciones de muestreo con trampas cámara en la Reserva Natural Chamanapamba y seis en el Bosque Protector Cerro La Candelaria. Estas dos áreas de conservación están ubicadas en la provincia de
Tungurahua, Ecuador, en la cuenca alta del río Pastaza en las estribaciones orientales de los Andes limitando con el Parque Nacional Sangay (Fig. 1). Las estaciones de muestreo estuvieron separadas en diferentes laderas dentro de las zonas de conservación, con trampas cámara ubicadas en transectos de $500 \mathrm{~m}$, una al inicio y otra al final. Las trampas cámara se situaron a $50 \mathrm{~cm}$ del suelo, en áreas previamente limpiadas para evitar fotos fantasmas, y trabajaron 24 horas/día durante 20 días/mes. Los registros aquí reportados se basan en fotografías y vídeos obtenidos con trampas-cámara Bushnell ${ }^{\circledR}$ 8MP Trophy Cam Estándar. Las coordenadas geográficas y elevación de cada estación se obtuvieron por medio de un GPS Garmin ${ }^{\circledR}$ 60CSx.

Entre diciembre 2011 y marzo 2012 obtuvimos fotografías de Eira barbara en cuatro estaciones de muestreo 


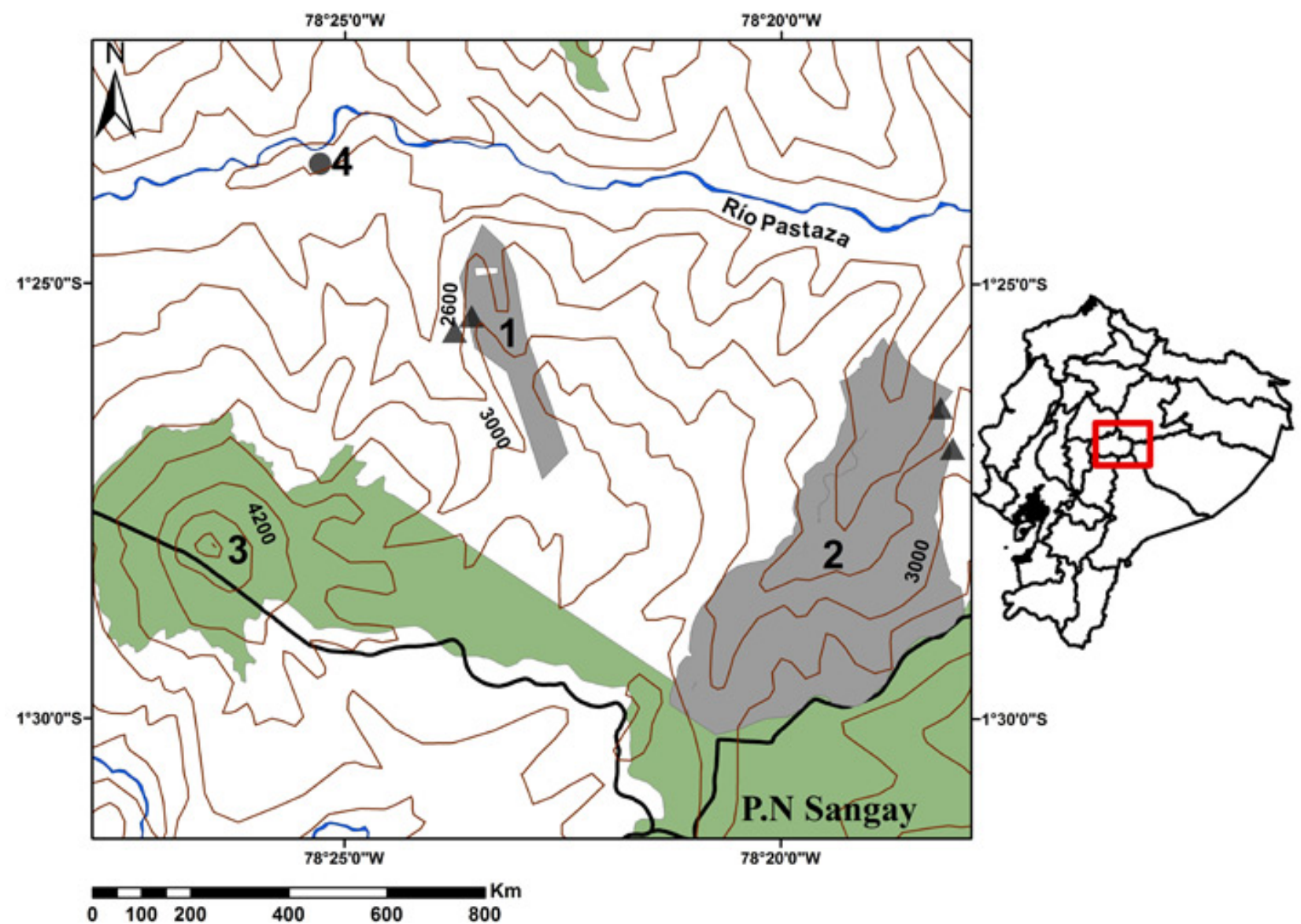

Figura 1. Localidades de Eira barbara en Ecuador que amplían su rango altitudinal sobre los $2600 \mathrm{~m}$, los triángulos representan las estaciones de trampas cámara en: 1) Reserva Natural Chamanapamba; 2) Bosque Protector Cerro La Candelaria. Puntos de referencia: 3) Volcán Tungurahua; 4) Baños de Agua Santa.

ubicadas en los bosques nublados de la Reserva Natural Chamanapamba (estaciones T.T.7 y T.T.8) y del Bosque Protector Cerro La Candelaria (estaciones T.6 y T.10) ubicadas entre 2665 y $3100 \mathrm{~m}$ de altitud (Tabla 1). Todos estos registros incrementan el rango altitudinal conocido para la especie. En particular, el registro dentro del bosque nublado de la Reserva Natural Chamanapamba que alcanza los 3100 metros de elevación.

Tanto la Reserva Natural Chamanapamba y el Bosque Protector Cerro La Candelaria constituyen amplios remanentes boscosos en buen estado de conservación, caracterizados por la presencia de árboles de Podocarpus, Myrcianthes, Clusia, gran cantidad de bromelias y Bryophytas. Su accidentada geografía con vertientes agrestes y pendientes de aproximadamente $85^{\circ}$, hacen de estas reservas áreas de refugio, paso y amortiguamiento para la mastofauna de la zona [8-9], que podría provenir del Parque Nacional Sangay. Del total de fotografías de mamíferos obtenidas, los registros de E. barbara representaron $1,2 \%$ en la Reserva Natural Chamanpamba y 22\% en el Bosque Protector Cerro La Candelaria.
No se ha documentado ampliamente los hábitos ecológicos de la especie, sin embargo, se presume que estos individuos realizan grandes desplazamientos en busca de recursos [3], habiéndose definido un área de vida de 530 ha. y estableciendo la utilización de varios tipos de hábitat disponibles para la especie [10]. Esta premisa permitiría suponer que E. barbara utiliza diferentes rutas en las zona de estudio como accesos a áreas con mayor disponibilidad de recursos, que corresponderían al Parque Nacional Sangay.

Durante las salidas de campo fue posible evidenciar los hábitos arborícolas y esquivos de la especie, observando a un individuo descender rápidamente de un árbol, al advertir la cercanía de personas (G. Ríos-Alvear, obs. pers.). Eira barbara exhibió comportamientos curiosos hacia las trampas cámara, destruyendo una de ellas. Se pudo identificar al agresor al revisar los videos, donde se observa como un individuo de E. barbara olfatea la cámara, muerde el lente y finalmente rasguña al sensor hasta romperlo por completo (Fig. 7).

Existe un espécimen de E. barbara depositado en el 
Tabla 1. Registros de Eira barbara sobre los 2600 metros de elevación

\begin{tabular}{|c|c|c|c|c|c|c|c|}
\hline Localidad & Reserva & Coordenadas & $\begin{array}{l}\text { Altitud } \\
\text { (m) }\end{array}$ & Cámara & Fecha & Hora & Fig. \\
\hline \multirow{13}{*}{$\begin{array}{l}\text { Tungurahua, Ba- } \\
\text { ños, Río Verde, } \\
\text { El Placer, Cerro } \\
\text { Candelaria }\end{array}$} & \multirow{13}{*}{$\begin{array}{c}\text { Bosque Protec- } \\
\text { tor Cerro la } \\
\text { Candelaria }\end{array}$} & $\begin{array}{l}1^{\circ} 26 ’ 25.46^{\prime \prime} \mathrm{S} ; \\
78^{\circ} 18^{\prime} 10.50 " \mathrm{O}\end{array}$ & 2708 & T.6 & 26/01/2012 & $16: 30$ & Fig. 4 \\
\hline & & \multirow{12}{*}{$\begin{array}{l}1^{\circ} 26^{\prime} 53.49 " ' \mathrm{~S} \\
78^{\circ} 18^{\prime} 2.32^{\prime \prime} \mathrm{O}\end{array}$} & \multirow{12}{*}{2766} & \multirow{12}{*}{ Т.10 } & $16 / 12 / 2011$ & $13: 59$ & \\
\hline & & & & & $17 / 12 / 2011$ & $14: 57$ & \\
\hline & & & & & $21 / 12 / 2011$ & $16: 11$ & \\
\hline & & & & & $04 / 01 / 2012$ & $12: 16$ & \\
\hline & & & & & $20 / 01 / 2012$ & $13: 17$ & Fig. 5 \\
\hline & & & & & $02 / 02 / 2012$ & $12: 18$ & \\
\hline & & & & & $20 / 02 / 2012$ & $9: 49$ & \\
\hline & & & & & $21 / 02 / 2012$ & $15: 43$ & \\
\hline & & & & & $25 / 02 / 2012$ & $18: 19$ & \\
\hline & & & & & $05 / 03 / 2012$ & $13: 03$ & \\
\hline & & & & & $12 / 03 / 2012$ & $16: 59$ & Fig. 6 \\
\hline & & & & & $13 / 03 / 2012$ & $9: 47$ & \\
\hline \multirow{3}{*}{$\begin{array}{l}\text { Tungurahua, } \\
\text { Baños, Ulba, } \\
\text { Chamanapamba }\end{array}$} & \multirow{3}{*}{$\begin{array}{l}\text { Reserva Natu- } \\
\text { ral Chamana- } \\
\text { pamba }\end{array}$} & \multirow{2}{*}{$\begin{array}{l}1^{\circ} 25^{\prime} 22.62{ }^{\prime \prime} \mathrm{S} \\
78^{\circ} 23^{\prime} 32.955^{\prime} \mathrm{O}\end{array}$} & \multirow{2}{*}{2665} & \multirow{2}{*}{ T.T.8 } & $09 / 12 / 2011$ & $9: 39$ & \\
\hline & & & & & $26 / 02 / 2012$ & $11: 38$ & Fig. 3 \\
\hline & & $\begin{array}{l}1^{\circ} 25^{\prime} 33.17^{\prime \prime} \mathrm{S} ; \\
78^{\circ} 23^{\prime} 44.60 " \mathrm{O}\end{array}$ & 3100 & T.T.7 & $01 / 10 / 2011$ & $6: 58$ & Fig. 2 \\
\hline
\end{tabular}

Field Museum de Chicago (FMNH 70767) colectado en el Río Balcones, municipio de Guasca, departamento de Cundinamarca, Colombia, por Phillip Hershkovitz a 2750 metros de elevación, el 29 de mayo de 1952. Este registro y la información del presente estudio sugieren que E. barbara habita sobre los 2500 metros de elevación al menos en Colombia y Ecuador, y que los estudios sobre su distribución no han sido exhaustivos. La utilización de nuevas metodologías como el fototrampeo

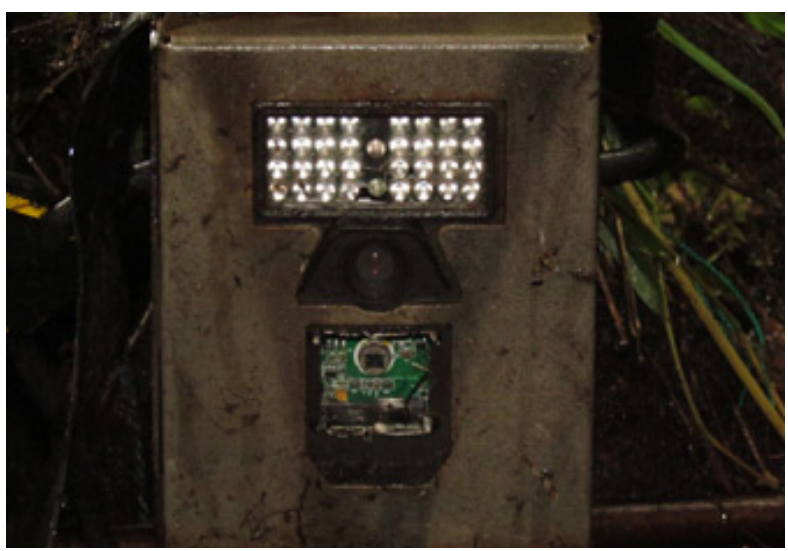

Figura 7. Cámara trampa destruida por Eira barbara en la estación T.10 del Bosque Protector Cerro La Candelaria, Tungurahua, Ecuador. pueden arrojar nueva información geográfica y comportamental de la especie. Consideramos necesario el monitoreo continuo con trampas cámara en rangos altitudinales superiores a los $2500 \mathrm{~m}$ para evaluar las poblaciones de E. barbara y sus patrones de desplazamiento.

\section{Agradecimientos}

Este estudio se realizó como parte de una investigación para la identificación de áreas prioritarias para la conservación del Tapir de Montaña Tapirus pinchaque y del Oso Andino Tremarctos ornatus, dentro del Proyecto de Conservación del Tapir de Montaña en la vertientes centrales del Corredor Llanganates-Sangay (PCTA), e iniciativas de conservación de la Fundación Oscar Efrén Reyes y la Fundación Ecominga. Por el apoyo en las salidas de campo y por su interés en la realización de proyectos de conservación, agradecemos especialmente a los guardaparques de Fundación Ecominga: Jesús, Fausto, Santiago y Luis Recalde; y a Manuel Chapungal por la ayuda brindada en campo dentro de la Reserva Natural Chamanapamba. Agradecemos también a dos revisores externos quienes aportaron con importantes sugerencias para el manuscrito. 


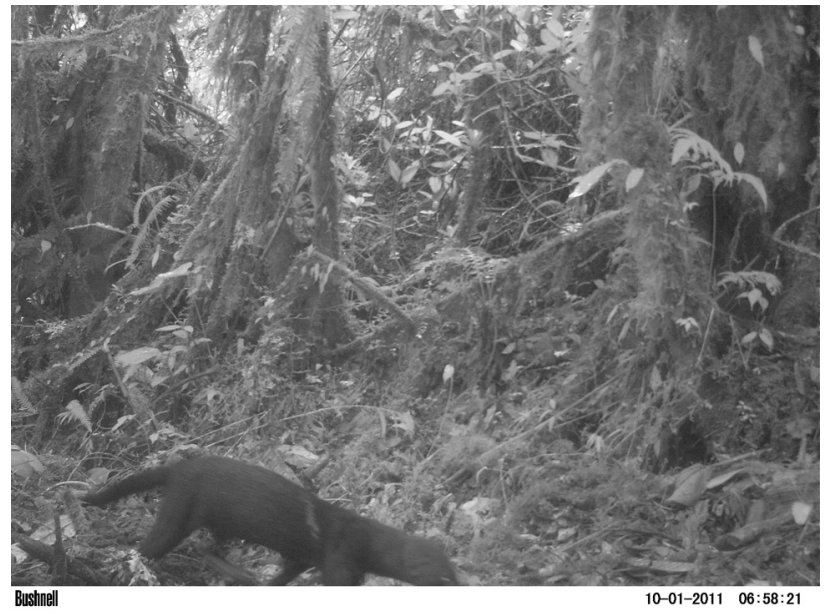

Figura 2. Registro de Eira barbara a $\mathbf{3 1 0 0}$ metros de elevación en la estación T.T.7 de la Reserva Natural Chamanapamba, Tungurahua, Ecuador.

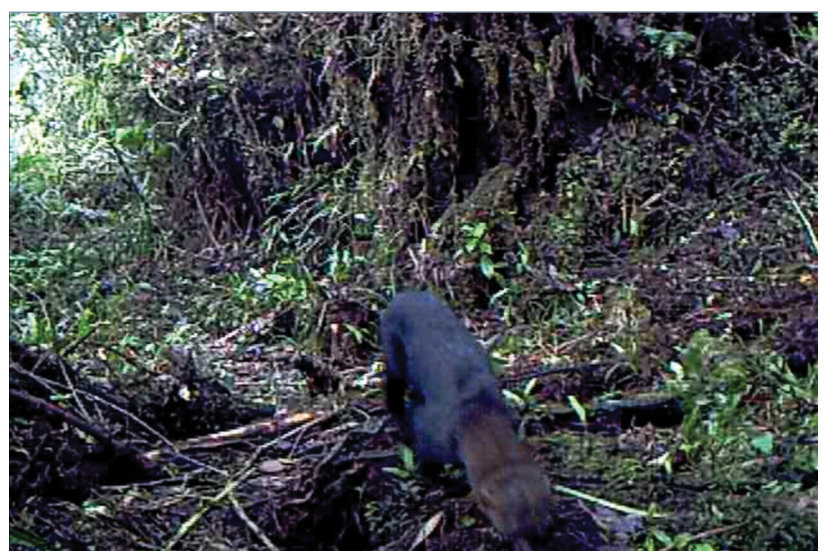

Figura 4. Registro de Eira barbara a 2708 metros de elevación en la estación T.6 del Bosque Protector Cerro La Candelaria, Tungurahua, Ecuador.

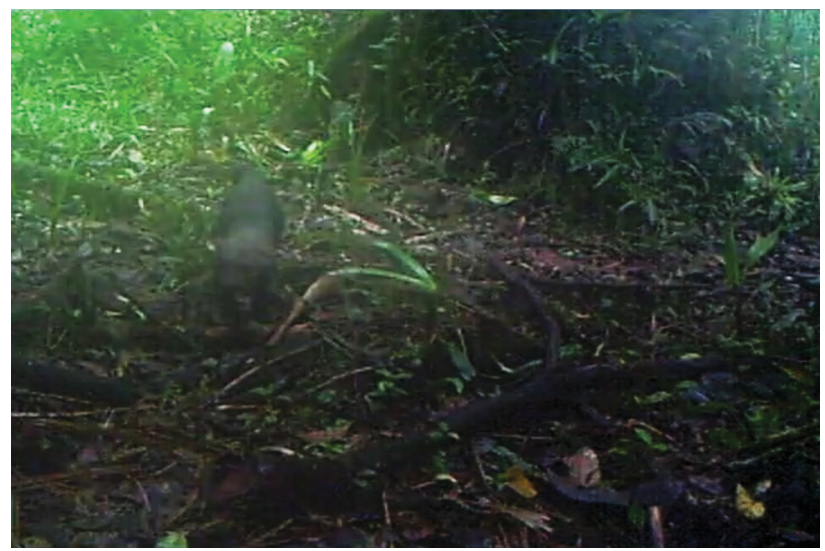

Figura 6. Registro de Eira barbara a 2766 metros de elevación en la estación T.10 del Bosque Protector Cerro La Candelaria, Tungurahua, Ecuador.

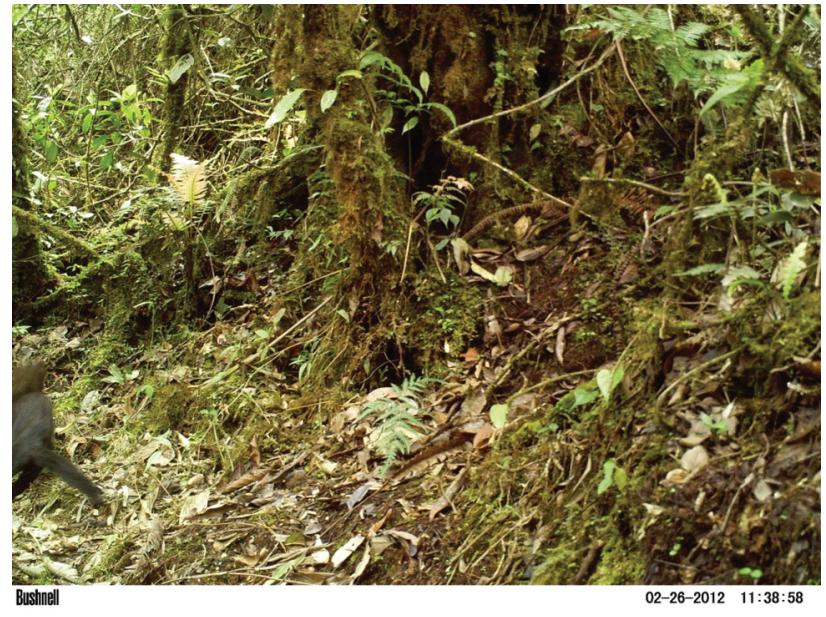

Figura 3. Registro de Eira barbara a 2665 metros de elevación en la estación T.T.8 de la Reserva Natural Chamanapamba, Tungurahua, Ecuador.

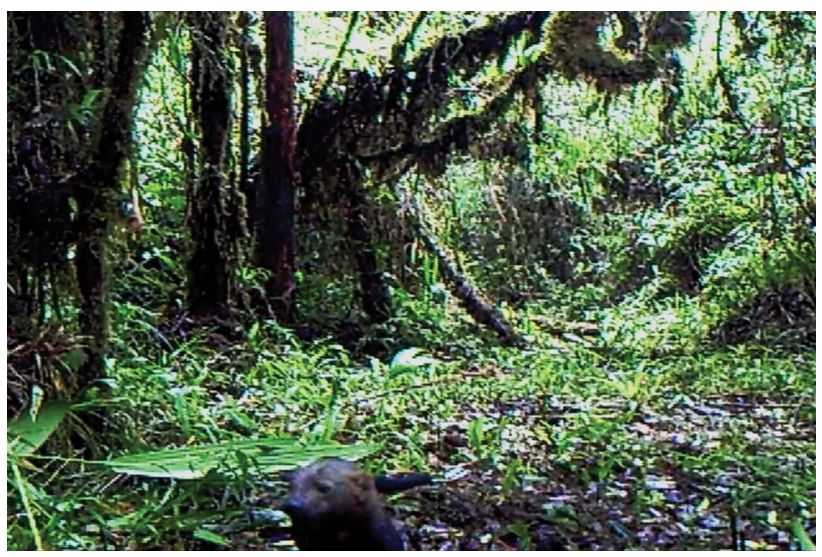

Figura 5. Registro de Eira barbara a 2766 metros de elevación en la estación T.10 del Bosque Protector Cerro La Candelaria, Tungurahua, Ecuador. 


\section{Referencias Bibliográficas}

[1] Wilson, D. E.; Reeder, D. M. (eds.). 2005. "Mammal species of the World, a taxonomic and geographic reference". 3ra Edición. Smithsonian Institution Press. American Society of Mammalogists. Washington D.C.

[2] Emmons, L. H.; Freer, F. 1990. "Neotropical rainforest mammals: a field guide". University of Chicago Press, Illinois.

[3] Presley, S. J. 2000. "Eira barbara". Mammalian Species, 636: 1-6.

[4] Eisenberg, J. F. 1989. "Mammals of the Neotropics: the northern Neotropics, Panama, Colombia, Venezuela, Guyana, Suriname, French Guiana". The University of Chicago Press: Illinois, EE.UU.

[5] Tirira, D. G. 2007. "Mamíferos del Ecuador. Guía de campo". Publicación Especial de los Mamíferos del Ecuador 6, Ediciones Murciélago Blanco: Quito, Ecuador.

[6] Tirira, D. G. 2014. "Mamíferos del Ecuador: diversidad". Versión 4. Enlace: www.mamiferosdelecuador.com. Grupo Murciélago Blanco: Quito, Ecuador. Fecha de consulta: 02 abril 2015.

[7] Dobbs, R.C.; Martin, P.R.; Kuehn, M.J. 2001. "On the nest, eggs, nestlings, and parental care of the Scaled Antpitta (Grallaria guatimalensis)". Ornitología Neotropical, 12: 225-233.

[8] Reyes-Puig, C.P.; Ríos-Alvear, G.D. 2013. "Monitoreo del tapir de montaña (Tapirus pinchaque) en el bosque nublado de la Reserva Natural Chamanapamba". Boletin Técnico 11, Serie Zoológica 8-9: 74-90

[9] Ríos-Alvear, G.D; Reyes-Puig, C.P. 2013. "Monitoreo del oso andino (Tremarctos ornatus) en tres áreas de bosque nublado en la cuenca alta del Pastaza". Boletín Técnico 11, Serie Zoológica 8-9: 91-108 